INTERVENTIONAL CARDIOLOGY AND SURGERY

\title{
Nicorandil versus isosorbide dinitrate as adjunctive treatment to direct balloon angioplasty in acute myocardial infarction
}

\author{
N Ikeda, T Yasu, N Kubo, S Hashimoto, Y Tsuruya, M Fujii, M Kawakami, M Saito
}

Heart 2004;90:181-185. doi: 10.1136/hrt.2003.013789

See end of article for authors' affiliations

Correspondence to:

Dr T Yasu, Department of Integrated Medicine I, Omiya Medical Centre, Jichi Medical School, 1-

847 Amanuma, Saitama, Saitama 330-8503,

Japan; tyasu@

omiya.jichi.ac.jp

Accepted 3 July 2003

\begin{abstract}
Objective: To compare the effects of nicorandil (a hybrid ATP sensitive potassium channel $\left(\mathrm{K}^{+}\right.$ATP channel) opener/nitric oxide donor) with those of isosorbide dinitrate (ISDN) on myocardial microcirculation and cardiac function in patients with acute myocardial infarction (AMI) who had undergone reperfusion treatment by direct balloon angioplasty.

Design: Double blind randomised study.

Patients: 60 patients with AMI in Killip class I.

Interventions: Patients were assigned into two treatment groups: a nicorandil group ( $\mathrm{n}=30$ ) and an ISDN group ( $\mathrm{n}=30$ ). Each drug was infused intravenously at $6 \mathrm{mg} / \mathrm{h}$ for 72 hours starting at admission and was administered directly to the treated coronary artery immediately after angioplasty.

Results: Compared with ISDN, nicorandil more frequently caused recovery of ST segment elevation just after reperfusion (15 of $27(55.5 \%)$ in the nicorandil group $\vee 5$ of 26 (19.2\%) in the ISDN group, $\mathrm{p}=0.006)$. The nicorandil group had higher values of averaged peak velocity 40 minutes after reperfusion (mean (SD) $24.8(13.3) \mathrm{cm} / \mathrm{s} v 16.0(11.1) \mathrm{cm} / \mathrm{s}, \mathrm{p}=0.045)$ and higher values of regional wall motion of the infarcted area three weeks after onset of AMI $(-1.78$ (1.11) v-2.50 (1.04) SD/chord, $\mathrm{p}=0.046)$.

Conclusions: A combination of nicorandil drip infusion starting before reperfusion and intracoronary injection immediately after reperfusion is more effective than a similarly performed infusion of ISDN in preserving myocardial microcirculation in the reperfused $\mathrm{AMI}$ area. The nicorandil regimen resulted in better left ventricular regional wall motion.
\end{abstract}

oronary reperfusion is now widely used to restore blood flow to the jeopardised myocardium in patients with acute myocardial infarction (AMI). However, several studies have shown that about $20 \%$ to $35 \%$ of angiographically successful recanalisations of an infarct related artery fail to salvage ischaemic myocardium. ${ }^{1-3}$ This unfavourable outcome is related to a discrepancy between an open epicardial coronary artery and the absence of blood flow (no reflow) in the damaged distal microvessels.

Nicorandil, a hybrid molecule comprising an ATP sensitive potassium channel $\left(\mathrm{K}^{+}{ }_{\text {ATP }}\right.$ channel) opener and a nitric oxide donor, has been approved as a treatment for angina pectoris in Japan and Europe. Recently, a large clinical trial, the IONA (impact of nicorandil on angina) study, ${ }^{4}$ showed that nicorandil reduces the frequency of cardiovascular events in patients with stable effort angina compared with placebo. Nicorandil promotes the recovery of post-ischaemic contractile dysfunction and reduces infarct size in animals. ${ }^{5-8}$ Numerous mechanisms for the salutary actions of nicorandil have been postulated, including reduction of preload and afterload, anti-free radical and neutrophil modulating properties, ${ }^{9-11}$ vasodilatation of small coronary arteries, and mimicking of ischaemic preconditioning. ${ }^{12}$ Nitric oxide donors such as isosorbide dinitrate (ISDN) also exert a cardiac protective action during ischaemia-reperfusion. ${ }^{13}{ }^{14}$ In previous studies, nicorandil improved functional and clinical outcomes compared with coronary reperfusion alone in patients with AMI. ${ }^{15-17}$ However, no double blinded trials have compared the effects of nicorandil and of ISDN in patients with AMI.

The aim of this study was to assess the effects of nicorandil on microcirculation, functional improvement, and in-hospital complications and to compare these effects with those of ISDN, a more potent nitric oxide donor. ${ }^{11}$ To assess myocardial perfusion, coronary flow velocity was measured with a Doppler guidewire.

\section{METHODS}

\section{Patient selection}

From October 1998 to March 2000, a total of 209 consecutive patients with AMI were admitted to our coronary care unit. Patients eligible for this study were men and women $<80$ years of age with a history of chest discomfort over 30 minutes in duration associated with ST segment elevation of more than $0.2 \mathrm{mV}$ in at least two contiguous leads. Maximum serum concentrations of creatine kinase more than twice the upper limit of normal and admission within 12 hours after symptom onset were required for inclusion. Patients were excluded if they were in Killip grade II or higher or had a history of AMI or prior coronary bypass surgery. Of these, 60 patients (42 men, 18 women; mean (SD) age 63 (9) years) who fulfilled the inclusion criteria were enrolled in the present study. These 60 patients were randomly assigned to two treatment groups in a double blind fashion: the nicorandil group $(\mathrm{n}=30)$ and the ISDN group $(\mathrm{n}=30)$. The study protocol was approved by the ethical committee of Jichi Medical School and written informed consent was obtained from each patient.

\footnotetext{
Abbreviations: AMI, acute myocardial infarction; IONA, impact of nicorandil on angina; ISDN, isosorbide dinitrate; $\mathrm{K}^{+}$ATP channel; ATP sensitive potassium channel; TIMI, thrombolysis in myocardial infarction
} 
Table 1 Clinical characteristics of the study patients

\begin{tabular}{llll}
\hline & Nicorandil $(\mathbf{n = 3 0})$ & ISDN (n=30) & p Value \\
\hline Mean age (years) & $60(9)$ & $63(10)$ & 0.38 \\
Male sex & $23 / 30(76.7 \%)$ & $25 / 30(83.3 \%)$ & 0.52 \\
Diabetes mellitus & $5 / 30(16.7 \%)$ & $6 / 30(20.0 \%)$ & 0.74 \\
Preinfarction angina (<48 h) & $14 / 30(33.3 \%)$ & $12 / 30(40.0 \%)$ & 0.80 \\
Mean time from onset to reperfusion (h) & $5.2(2.5)$ & $5.7(3.3)$ & 0.47 \\
Anterior MI & $10 / 30(33.3 \%)$ & $12 / 30(40.0 \%)$ & 0.59 \\
Non-Q MI & $2 / 30(6.6 \%)$ & $1 / 30(3.3 \%)$ & 0.59 \\
TIMl grade (0, 1) before angioplasty & $(26,4)$ & $(25,5)$ & 0.72 \\
TIMl grade (2, 3) after angioplasty & $(1,29)$ & $(3,27)$ & 0.30 \\
Collateral (grade $>2)$ & $3 / 30(10.0 \%)$ & $2 / 30(6.7 \%)$ & 0.64 \\
ACE inhibitor & $14 / 30(46.7 \%)$ & $15 / 30(50.0 \%)$ & 0.79 \\
$\beta$ Blocker & $5 / 30(16.6 \%)$ & $7 / 30(23.3 \%)$ & 0.52 \\
Mean BP (mm Hg) & $92(9)$ & $89(7)$ & 0.33 \\
Mean HR (beats/min) & $79(11)$ & $78(12)$ & 0.96 \\
Use of stent & $1 / 30(70.0 \%)$ & $19 / 30(63.3 \%)$ & 0.58 \\
Maximum CK concentrations (iu/l) & $2953(1950)$ & $3595(2274)$ & 0.25 \\
\hline Values are mean (SD) or number (\%). & & & \\
ACE, angiotensin converting enzyme; BP, blood pressure; CK, creatine kinase; HR, heart rate; ISDN, isosorbide \\
dinitrate; Ml, myocardial infarction; TIMl, thrombolysis in myocardial infarction. & \\
\hline
\end{tabular}

\section{Study design}

This study was designed as a prospective, double blind, randomised trial in a single centre. One of the investigators supervised the double blind study as a safety and data monitor.

\section{Protocol}

Immediately after diagnosis of AMI, the study drug (nicorandil or ISDN) was infused intravenously at $6 \mathrm{mg} / \mathrm{h}$ for 72 hours. All patients underwent emergent cardiac catheterisation through a femoral artery after injection of $3000 \mathrm{U}$ of heparin. Each patient received $162 \mathrm{mg}$ of aspirin and an additional $7000 \mathrm{U}$ of heparin before angioplasty. Stents were implanted if unsatisfactory angioplasty results were obtained. Stented patients received ticlopidine (200 mg twice a day) and aspirin (81 mg), whereas non-stented patients were given aspirin only. Successful reperfusion was defined as thrombolysis in myocardial infarction (TIMI) grade 2 or higher, as determined by angiography. Immediately after confirmation of successful coronary reflow, the study drug $(2 \mathrm{mg})$ was administered into the treated coronary artery. We used a combination of intravenous drip infusion starting at the time of diagnosis of AMI

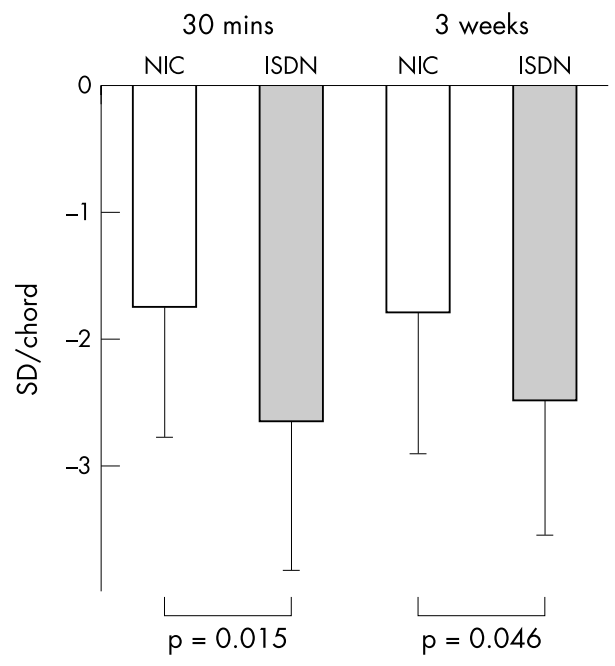

Figure 1 Regional wall motion was analysed by the centre line method and expressed as SD/chord. SD/chord 30 minutes after reperfusion and three weeks after onset was significantly lower in the nicorandil (NIC) group than in the isosorbide dinitrate (ISDN) group. and intracoronary artery infusion immediately after angioplasty to elicit maximal effects of the study drugs as adjunctive treatments. Because abrupt activation of $\mathrm{K}^{+}{ }_{\text {ATP }}$ channels has been shown to be proarrhythmic, ${ }^{18}$ we slowly injected both drugs into the treated coronary artery over a period of 60 seconds. Heparin infusion was continued for 72 hours and systolic blood pressure was kept below $150 \mathrm{~mm} \mathrm{Hg}$ by intravenous drip infusion of nicardipine after angioplasty. Systemic blood pressure and the pulse in the left arm were measured by specially trained nurses at 7 am, noon, $5 \mathrm{pm}$, and $9 \mathrm{pm}$ every day during the first seven days. The results of all 28 measurements were averaged. Serum concentrations of creatine kinase were measured every four hours from admission until they peaked and began to fall. The decision of whether to administer angiotensin converting enzyme inhibitors and $\beta$ blockers was left to the attending physician. Cardiac catheterisation was repeated three weeks after onset of AMI.

\section{Outcome assessment}

The total ST segment elevation excluding aVR (defined as $\sum \mathrm{ST}$ ) was measured when TIMI 0 or 1 flow was confirmed before reperfusion. The $\sum$ ST was also examined when TIMI 2 or 3 flow was confirmed for the first time after reperfusion by angioplasty. The isoelectric line was selected at the level of the PQ junction and ST segment elevation was measured at the $J$ point. Posterior and lateral AMIs $(n=7)$ were excluded from the electrocardiographic analysis. The change in $\sum \mathrm{ST}$ at reperfusion was classified into one of the following two groups according to degree: decrease of $\geqslant 20 \%$ (ST recovered); and decrease of $<20 \%$ or increase (ST not recovered) as described previously. ${ }^{19}$

The status of the collateral circulation was defined as reported by Rentrop and colleagues. ${ }^{20}$ Left ventriculography was performed about 30 minutes (21-40 minutes) after the reperfusion and three weeks after the onset of AMI. One observer without knowledge of the patients' data carefully traced all left ventricular end diastolic and end systolic endocardial contours. Left ventricular end diastolic volume index and end systolic volume index were determined. Global left ventricular ejection fraction was determined by the arealength method. Regional left ventricular wall motion was measured by the centre line method and expressed as SD/ chord, as described elsewhere. ${ }^{21}{ }^{22}$ Posterior and lateral AMIs were excluded from regional wall motion analysis.

Coronary blood flow velocity was measured about 40 minutes (31-60 minutes) after the reperfusion with a 
EDVI

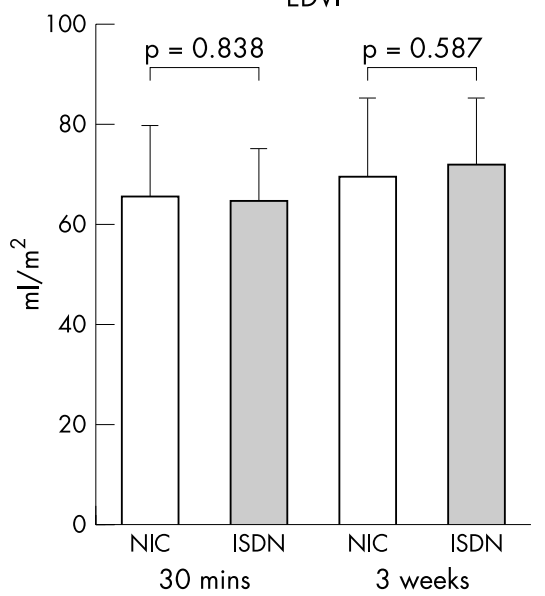

ESVI

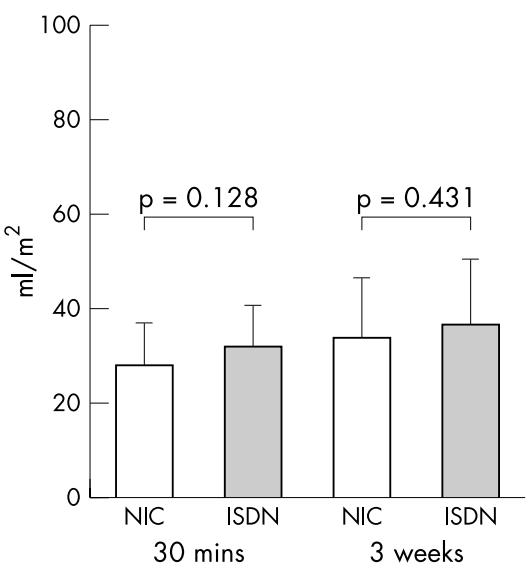

EF

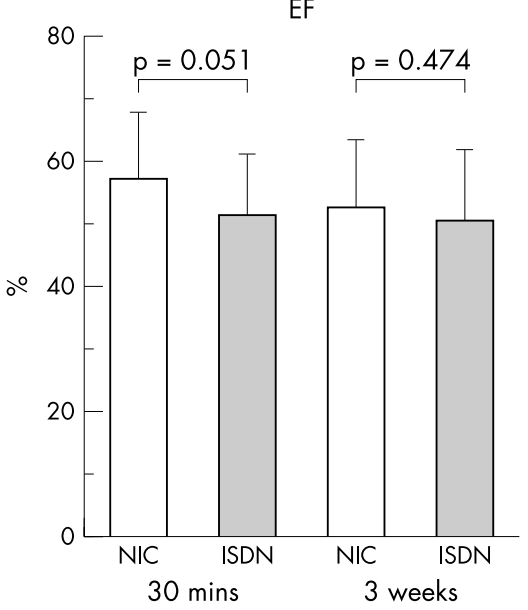

Figure 2 Left ventricular volume (EDVI and ESVI) and global ejection fraction (EF) in left ventriculography are shown. There were no differences in left ventricular volume between the two treatment groups.
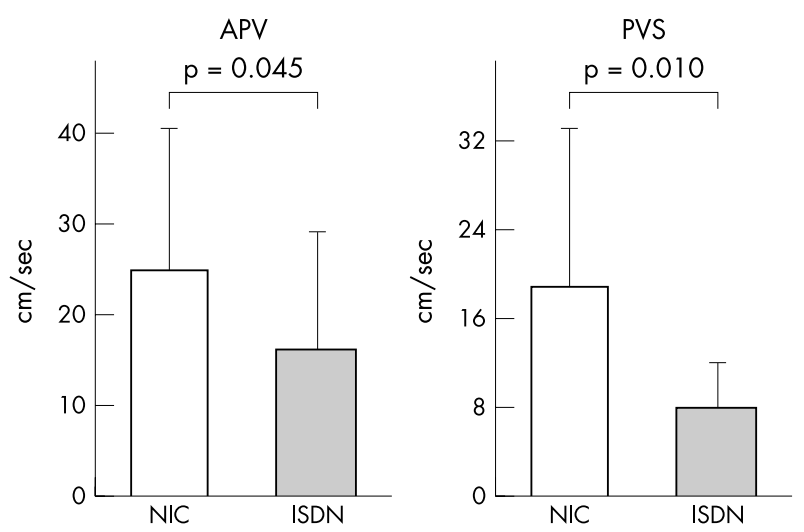

Doppler guidewire connected to a real time spectrum analyser, as described elsewhere. ${ }^{23}$ Time averaged peak velocity was computed on line and continuously recorded on videotape. The peak systolic antegrade flow velocity and frequency of early systolic retrograde flow were also analysed.

\section{Statistical analysis}

Data are presented as mean (SD). Groups were compared with Student's $t$ test or analysis of variance with Fisher's post hoc test for continuous variables and $\chi^{2}$ test for categorical values, as appropriate. A probability value of $\mathrm{p}<0.05$ was considered to indicate significance.

\section{RESULTS}

\section{Baseline characteristics}

There were no significant differences between the nicorandil and ISDN group with regard to age, sex, diabetes, preinfarction angina, infarction site, TIMI grade before and after angioplasty, collateral grade, time from onset to reperfusion, blood pressure, heart rate, use of stents, administration of angiotensin converting enzyme inhibitors and $\beta$ blockers, or maximum serum concentrations of creatine kinase (table 1).

\section{Electrocardiogram}

Nicorandil more frequently caused recovery of ST segment elevation just after reperfusion (15 of $27(55.5 \%))$ than did ISDN ( 5 of $26(19.2 \%), p=0.006)$.

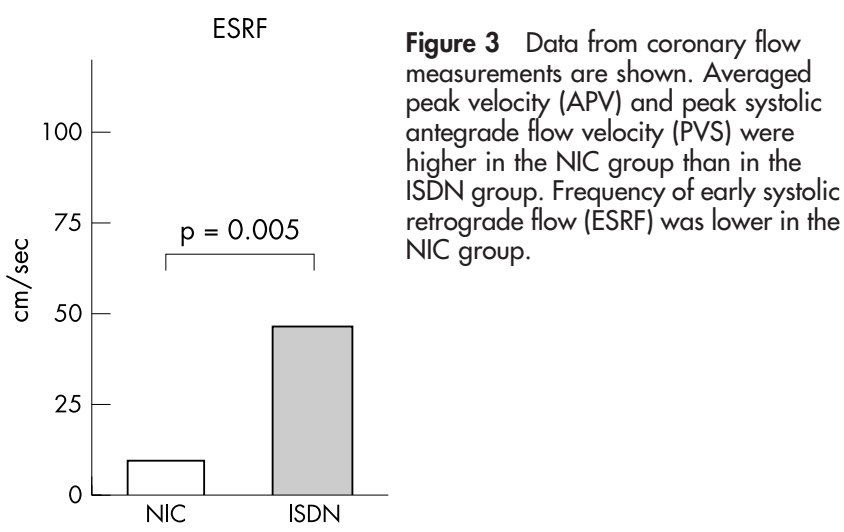

\section{Left ventriculography}

Regional wall motion of the infarct area 30 minutes after reperfusion was significantly greater in the nicorandil group than in the ISDN group (mean (SD) -1.75 (1.03) $v-2.66$ (1.16) SD/chord, $p=0.015)$. This effect continued for the three weeks after the onset of AMI $(-1.78$ (1.11) $v-2.50$ (1.04) SD/chord, $p=0.046$ ) (fig l). There were no differences in left ventricular volume between the two treatment groups (fig 2).

\section{Coronary flow measurements}

Of the coronary flow velocity variables measured 40 minutes after reperfusion, averaged peak velocity $(24.8$ (13.3) $v 16.0$ (11.1) $\mathrm{cm} / \mathrm{s}, \mathrm{p}=0.045)$ and peak systolic antegrade flow velocity (18.8 (12.9) $v$ $7.9 \quad(4.2) \mathrm{cm} / \mathrm{s}, \quad \mathrm{p}=0.010)$ were significantly higher in the nicorandil group than in the ISDN group (fig 3). The frequency of early systolic retrograde flow was significantly lower in the nicorandil group (4 of 30 $(13.3 \%) v 14$ of $30(46.7 \%), p=0.005)$ (fig 3 ).

Table 2 Major complications

\begin{tabular}{llll}
\hline & $\begin{array}{l}\text { Nicorandil } \\
(\mathbf{n}=30)\end{array}$ & ISDN $(\mathbf{n}=30)$ & p Value \\
\hline Sustained VT & & & \\
(rate $>100$ beats $/ \mathrm{min})$ & $1 / 30(3.3 \%)$ & $2 / 30(6.7 \%)$ & 0.55 \\
Pericardial effusion & $3 / 30(10 \%)$ & $5 / 30(16.6 \%)$ & 0.25 \\
Ventricular rupture & $0 / 30(0 \%)$ & $1 / 30(3.3 \%)$ & 0.32 \\
\hline VT, ventricular tachycardia. & & \\
\hline
\end{tabular}




\section{Complications}

After the intracoronary injection of nicorandil, slow ventricular tachycardia (rate $<100$ beats/min, also known as accelerated idioventricular beats) appeared in three patients but did not cause deterioration of the circulatory condition. There was no significant difference in the number of patients with sustained ventricular tachycardia (rate $\geqslant 100$ beats/ min), which required direct current cardioversion. Ventricular fibrillation was not seen in any patient during the study period. There was no significant difference in the incidence of pericardial effusion between the treatment groups. One patient in the ISDN group had a ventricular free wall rupture. He underwent an urgent surgical repair and was discharged (table 2). There were no deaths during the study period. Patients did not experience congestive heart failure or post-infarction angina during hospitalisation.

\section{DISCUSSION}

This double blind trial showed nicorandil to be more effective than ISDN in preserving left ventricular regional function at three weeks after AMI.

Nicorandil more frequently caused recovery of ST segment elevation just after reperfusion. We previously reported ST segment elevation after reperfusion as an index for predicting improvement of left ventricular systolic function in patients with reperfused anterior AMI. ${ }^{19}$ There was no difference between the treatment groups in regard to time from onset to reperfusion, existence of preinfarction angina, or grade of collateral vessels. Regional wall motion of the infarcted area at 30 minutes after reperfusion was higher in the nicorandil group than in the ISDN group, and this effect continued for the three weeks after the onset of AMI. This suggests that nicorandil mimics ischaemic preconditioning or suppresses reperfusion injury involving reversible ischaemia-reperfusion injury ("stunned myocardium").

Restoration of myocardial perfusion immediately after coronary revascularisation is an important mechanism for salvaging the post-ischaemic myocardium. ${ }^{2}$ According to a double blind study of 300 patients with AMI who underwent coronary angiography, abciximab, a glycoprotein IIb/IIIa antagonist, plus stenting improved the primary end point (a composite of death, reinfarction, or urgent revascularisation of the target vessel), TIMI flow classification before and after stenting, and left ventricular function six months after onset compared with placebo plus stenting. ${ }^{24}$ Abciximab has not been approved in Japan yet and the cost effectiveness of this drug requires further study before it can be used routinely. ${ }^{25}$ In previous studies, salutary actions of nicorandil on the coronary microvasculature were assessed by myocardial contrast echocardiography. ${ }^{15} 16$ In the present study, coronary flow velocity was measured with a Doppler guidewire to assess myocardial perfusion. Iwakura and colleagues ${ }^{23}$ found a strong correlation between no reflow (shown by myocardial contrast echocardiography) and early systolic retrograde flow (measured by Doppler guidewire). The nicorandil group had a higher coronary flow velocity after reperfusion and a lower frequency of early systolic retrograde flow than the ISDN group. Dilatation of smaller arterioles is an important factor in the extent of myocardial stunning, ${ }^{26} 27$ infarct size, and functional recovery ${ }^{28}$ after ischaemiareperfusion. $\mathrm{K}^{+}{ }_{\text {ATP }}$ channel activators dilate resistant vessels (especially arterioles with diameters $<100 \mu \mathrm{m})^{30} 31$ and increase collateral blood flow. ${ }^{32}{ }^{33}$ In contrast, pure nitric oxide donors such as ISDN do not dilate small arteries and arterioles with diameters $<100 \mu \mathrm{m}$. Leucocytes are key players in the aggravation of post-reperfusion microcirculation through mechanisms such as spasm of microvessels, leucocyte plugging in microvessels, and production of oxyradical mediators. ${ }^{34} 35$ In a recent study we conducted with in vivo and in vitro methods, ${ }^{14}$ nicorandil and a specific mitochondrial $\mathrm{K}_{\text {ATP }}^{+}$channel opener (diazoxide) suppressed the following processes of leucocyte activation: leucocyte pseudopod formation in the presence of $N$-formyl-methionyl-leucylphenylalanine under shear stress; adhesion of leucocytes to postcapillary venular endothelial cells; and extravascular migration of leucocytes in rat mesenteric microvessels subjected to ischaemia-reperfusion. An increase in shear rate caused by increased microvessel flow induced by a $\mathrm{K}_{\text {ATP }}^{+}$channel activator may also reduce neutrophil recruitment ${ }^{36}$ because leucocyte attachment to and migration through inflamed vessels is somewhat dependent on shear rate. In addition, Gross et $a l^{9}$ and Pieper et $a l^{10}$ found that the beneficial effects of nicorandil in recovery from ischaemiareperfusion injury may be due partly to inhibition of free radical production by leucocytes.

In a recent large clinical trial $(\mathrm{n}=5126),{ }^{4}$ nicorandil significantly reduced the number of cardiovascular events in patients with stable effort angina compared with placebo (relative risk reduction $15 \%, \mathrm{p}=0.025$ ) during a follow up period of up to three years. This supports the benefit of pharmacological preconditioning in the management of stable angina. In addition, recent experiments ${ }^{37} 38$ with knockout mice have clearly documented that $\mathrm{K}^{+}{ }_{\text {ATP }}$ channel subunits Kir 6.2 and sulfonylurea receptor 2 have important roles in preventing fatal vasospastic angina.

\section{Conclusion}

Nicorandil administered in a combination of drip infusion starting during the ischaemic period and intracoronary injection immediately after reperfusion was more effective than a similarly performed infusion of ISDN in preserving the myocardial microcirculation in relatively low risk patients with AMI. The result was improved left ventricular wall motion in the infarction zone. The present results suggest the need for a large clinical trial to determine whether nicorandil should be recommended for patients with AMI.

\section{Authors' affiliations}

N Ikeda, T Yasu, N Kubo, S Hashimoto, Y Tsuruya, M Fujii,

M Kawakami, M Saito, Department of Integrated Medicine I, Omiya Medical Centre, Jichi Medical School, 1-847 Amanuma, Saitama, Saitama 330-8305, Japan

An abstract of this work was presented at the 50th annual meeting of the American College of Cardiology, 18 March 2001, Orlando, Florida.

\section{REFERENCES}

1 Schofer J, Montz R, Mathey DG. Scintigraphic evidence of the "no reflow" phenomenon in human beings after coronary thrombolysis. J Am Coll Cardiol 1985:5:593-8.

2 Ito $\mathrm{H}$, Tomooka T, Sakai N, et al. Lack of myocardial perfusion immediately after successful thrombolysis: a predictor of poor recovery of left ventricular function in anterior myocardial infarction. Circulation 1992;85:1699-705.

3 Ragosta M, Camarano G, Kaul S, et al. Microvascular integrity indicates myocellular viability in patients with recent myocardial infarction: new insights using myocardial contrast echocardiography. Circulation 1994:89.2562-9.

4 Anon. Effect of nicorandil on coronary events in patients with stable angina: the impact of nicorandil in angina (IONA) randomized trial. The IONA study group. Lancet 2002;359:1269-75.

5 Auchampach JA Cavero I, Gross GJ. Nicorandil attenuates myocardial dysfunction associated with transient ischemia by opening ATP-dependent potassium channels. J Cardiovasc Pharmacol 1992;20:765-71.

6 Mizumura T, Nithipatikom K, Gross GJ. Effects of nicorandil and glyceryl trinitrate on infarct size, adenosine release, and neutrophil infiltration in the dog. Cardiovasc Res 1995;29:482-9.

7 Klein HH, Pich S, Lindert-Heimberg S, et al. Comparative study on the effects of intracoronary nicorandil and nitroglycerin in ischemic, reperfused porcine hearts. Eur Heart J 1995;16:603-9.

8 Galie N, Guarnieri C, Ussia GP, et al. Limitation of myocardial infarct size by nicorandil after sustained ischemia in pigs. J Cardiovasc Pharmacol 1995;26:477-84.

9 Gross GJ, Pieper GM, Farber NE. Effects of nicorandil on coronary circulation and myocardial ischemia. Am J Cardiol 1989;63:11J-7J.

10 Pieper GM, Gross GJ. Anti-free radical and neutrophil-modulating properties of the nitrovasodilator, nicorandil. Cardiovasc Drugs Ther 1992;2:225-32. 
11 Yasu T, Ikeda N, Ishizuka N, et al. Nicorandil and leukocyte activation. J Cardiovasc Pharmacol 2002;40:684-92.

12 Kitakaze M, Minamino T, Node K, et al. Role of activation of 5-nucleotidase in the cardioprotection mediated by opening $\mathrm{K}^{+}$channels. Am J Physiol 1996;270:H1744-56

13 Kosonen O, Kankaanranta H, Malo-Ranta U, et al. Nitric oxide-releasing compounds inhibit neutrophil adhesion to endothelial cells. Eur J Pharmaco 1999:382:111-7.

14 Leesar MA, Stoddard MF, Dawn B, et al. Delayed preconditioning-mimetic action of nitroglycerin in patients undergoing coronary angioplasty. Circulation 2001; 103:2935-41.

15 Ito $\mathbf{H}$, Taniyama $Y$, Iwakura $\mathrm{K}$, et al. Intravenous nicorandil can preserve microvascular integrity and myocardial viability in patients with reperfused anterior wall myocardial infarction. J Am Coll Cardiol 1999;33:654-60.

16 Sakata Y, Kodama K, Komamura K, et al. Salutary effect of adjunctive intracoronary nicorandil administration on restoration of myocardial blood flow and functional improvement in patients with acute myocardial infarction. Am Heart J 1997; 133:616-21.

17 Kobayashi Y, Goto Y, Daikoku S, et al. Cardioprotective effect of intravenous nicorandil in patients with successful reperfusion for acute myocardial infarction. Jpn Circ J 1998;62:183-9.

18 Kempsford RD, Hawgood BJ. Assessment of the antiarrhythmic activity of nicorandil during myocardial ischemia and reperfusion. Eur J Pharmacol 1989;163:61-8.

19 Kobayashi N, Ohmura N, Nakada I, et al. Further ST elevation at reperfusion by direct percutaneous transluminal coronary angioplasty predicts poor recovery of left ventricular systolic function in anterior wall AMI. Am J Cardiol 1997;79:862-6.

20 Rentrop KP, Cohen M, Blanke $\mathrm{H}$, et al. Changes in collateral channel filling immediately after controlled coronary artery occlusion by an angioplasty balloon in human subjects. J Am Coll Cardiol 1985;5:587-92.

21 Sheehan FH, Bolson EL, Dodge HT, et al. Advantages and applications of the centerline method for characterizing regional ventricular function. Circulation 1986;74:293-305.

22 Kubo N, Ohmura N, Nakada l, et al. Exercise training induces left ventricular enlargement in patients with left ventricular dysfunction. Circulation 1997; $96: 1345$.

23 Iwakura K, Ito $\mathrm{H}$, Takiuchi S, et al. Alternation in the coronary blood flow velocity pattern in patients with no reflow and reperfused acute myocardial infarction. Circulation 1996;94:1269-75.
24 Montalescot G, Barragan P, Wittenberg O, et al. Platelet glycoprotein Ilb/Illa inhibition with coronary stenting for acute myocardial infarction. N Engl J Med 2001;344:1895-903

25 Stone GW, Grines CL, Cox DA, et al. Comparison of angioplasty with stenting, with or without abciximab, in acute myocardial infarction. N Engl J Med 2002;346:957-66.

26 Farber NE, Pieper GM, Gross GJ. Postischemic recovery in the stunned myocardium after reperfusion in the presence or absence of a flow-limiting coronary artery stenosis. Am Heart J 1988;116:407-20.

27 Stahl LD, Aversano TR, Becker LC. Selective enhancement of function of stunned myocardium by increased flow. Circulation 1986;74:843-51.

28 Agati L, Voci P, Bilotta F, et al. Influence of residual perfusion within the infarct zone on the natural history of left ventricular dysfunction after acute myocardial infarction: a myocardial contrast echocardiographic study. J Am Coll Cardiol 1994;24:336-42.

29 Camarano G, Ragosta M, Gimple LW, et al. Identification of viable myocardium with contrast echocardiography in patients with poor left ventricular systolic function caused by recent or remote myocardial infarction. Am J Cardiol 1995;75:215-9.

30 Komaru T, Lamping KG, Eastham CL, et al. Roles of ATP-sensitive potassium channels in coronary microvascular autoregulatory responses. Circ Res 1991;69:1146-51.

31 Nelson MT, Quayle JM. Physiological roles and properties of potassium channels in arterial smooth muscle. Am J Physiol 1995;268:C799-822.

32 Richer C, Pratz J, Mulder $\mathrm{P}$, et al. Cardiovascular and biological effects of $\mathrm{K}^{+}$ channel openers, a class of drugs with vasorelaxant and cardioprotective properties. Life Sci 1990;47:1693-705.

33 Maruyama M, Farber N, Gross GJ. Effect of the new potassium channel opener EMD 52892 on coronary collateral blood flow in dogs. FASEB J 1989;3:A897.

34 Romson JL, Hook BG, Kunkel SL, et al. Reduction of the extent of ischemic myocardial injury by neutrophil depletion in the dog. Circulation 1983;67:1016-23.

35 Hansen PR. Role of neutrophils in myocardial ischemia and reperfusion. Circulation 1995;91:1872-85

36 Fukuda S, Yasu T, Predescu DN, et al. Mechanism for regulation of fluid shear stress response in circulating leukocytes. Circ Res 2000;86:E13-8.

37 Miki T, Suzuki M, Shibasaki T, et al. Mouse model of Prinzmetal angina by disruption of the inward rectifier Kir 6.1. Nat Med 2002;8:466-72.

38 Chutkow WA, Pu J, Wheeler MT, et al. Episodic coronary artery vasospasm and hypertension develop in the absence of Sur2 K $\mathrm{K}_{\text {ATP }}$ channels. J Clin Invest 2002;110:203-8.

\section{FROM BMJ JOURNALS \\ Prognosis of angina with and without a diagnosis: 11 year follow up in the Whitehall II prospective cohort study}

Please visit the Heart website [www.heartinl. com] for a link to the full text of this article.
Harry Hemingway, Martin Shipley, Annie Britton, Michael Page, Peter Macfarlane, Michael Marmot

Objective: To investigate the prognosis of angina among people with and without diagnosis by a doctor and an abnormal cardiovascular test result.

Design: Prospective cohort study with a median follow up of 11 years.

Setting: 20 civil service departments originally located in London.

Participants: 10308 civil servants aged 35-55 years at baseline.

Main outcome measures: Recurrent reports of angina; quality of life (SF-36 physical functioning); non-fatal myocardial infarction; death from any cause $(\mathrm{n}=344)$.

Results: 1158 (11.4\%) participants developed angina, and 813 (70\%) had no evidence of diagnosis by a doctor at the time of the initial report. Participants without a diagnosis had an increased risk of impaired physical functioning (age and sex adjusted odds ratio of 2.36 (95\% confidence interval 1.91 to 2.90)) compared with those who had neither angina nor myocardial infarction throughout follow up. Among reported cases of angina without a diagnosis, the $15.5 \%$ with an abnormality on a study electrocardiogram had an increased risk of death (hazard ratio 2.37 ( 1.16 to 4.87 )). These effects were similar in magnitude to those in participants with a diagnosis of angina.

Conclusion: Undiagnosed angina was common and had an adverse impact on prognosis comparable to that of diagnosed angina, particularly among people with electrocardiographic abnormalities. Efforts to improve prognosis among people with angina should take account of this submerged clinical iceberg.

A BMJ 2003;327:895-898 